UVX 2010 (2011) 123-129

DOI: $10.1051 / \mathrm{uvx} / 2011017$

(C) Owned by the authors, published by EDP Sciences, 2011

\title{
Détecteurs UV pour le spatial : revue des besoins et de l'état de l'art
}

\author{
A. Penquer \\ CNES, 18 Av. Edouard Belin, 31401 Toulouse Cedex 9, France
}

\begin{abstract}
Résumé. Cet article fait le point dans un premier temps sur les problématiques de la détection UV dans le cadre des missions spatiales et sur les besoins qui en découlent en terme de détecteurs. Les différentes technologies existantes sont ensuite présentées. Pour finir, deux réalisations récentes de détecteurs menées dans le cadre d'études initiées par le CNES (Centre National d'Etudes Spatiales) seront décrites.
\end{abstract}

\section{MISSIONS SPATIALES DANS L'UV : BESOINS ET PROBLÉMATIQUES EN TERME DE DÉTECTION}

\subsection{Missions spatiales dans l'UV}

Les missions spatiales de détection dans l'UV (de 10 à $350 \mathrm{~nm}$ ) couvrent un large domaine d'applications. On rencontre en premier lieu l'étude du Soleil avec des imageurs (couronne et disque solaire) et des spectromètres. Les imageurs solaires ont pour but d'étudier divers phénomènes (éruptions solaires, phénomènes de «Coronal mass Ejection», vent solaire...) et de permettre de mieux comprendre leurs mécanismes et interactions. La principale mission en cours d'étude dans ce domaine est SOLAR ORBITER, menée par l'ESA. Ensuite, de nombreux spectromètres UV peuvent être rencontrés en planétologie dans le cadre de l'étude de la chimie atmosphérique. Les instruments concernés sont des spectromètres voire des spectro-imageurs dont le but est d'observer des raies particulières avec une bonne résolution (de l'ordre de $1 \mathrm{~nm}$ FWHM) dans des bandes spectrales dont la largeur varie entre quelques dizaines et quelques centaines de $\mathrm{nm}$, et ce afin d'étudier les compositions chimiques de l'atmosphère des planètes étudiées. On peut citer deux missions dans ce domaine : l'instrument PHEBUS de BEPI COLOMBO (ESA, étude de Mercure) et EJSM (ESA, NASA et JAXA, étude de Jupiter). Pour finir, les détecteurs UV présentent également un grand intérêt dans le domaine de l'exploration extragalactique. En effet, l'étude des baryons par spectro-imagerie présente un fort intérêt dans ce domaine de longueur d'onde car le contraste y est particulièrement bon (luminance résiduelle parasite faible dans l'UV).

\subsection{Besoins en terme de détection et problématiques associées}

Le besoin d'avoir un bon rendement de détection et un faible bruit est une constante dans tous les domaines d'application précédemment cités. En effet, les flux utiles sont en général faibles. Cela pose un problème majeur, puisque les détecteurs classiques à base de Silicium (CCD, APS) ont un rendement quantique très faible dans l'UV. En effet, le coefficient d'absorption est augmenté d'une décade entre 100 et $300 \mathrm{~nm}$ dans le Silicium, de sorte que les photons ne peuvent pas pénétrer suffisamment profondément pour atteindre la zone active, le photoélectron créé n'étant ainsi pas collecté. De plus, les flux parasites (lumière visible) peuvent s'avérer élevés surtout en observation solaire. Dans ce cas,

This is an Open Access article distributed under the terms of the Creative Commons Attribution-Noncommercial License 3.0, which permits unrestricted use, distribution, and reproduction in any noncommercial medium, provided the original work is properly cited. 
il est nécessaire d'utiliser des détecteurs insensibles à la lumière visible (ce qui n'est pas le cas des détecteurs classiques) ou des filtres. Ces filtres supplémentaires, outre des aspects qualité contraignants vis-à-vis d'une utilisation spatiale, présentent l'inconvénient de réduire de manière non négligeable les flux utiles déjà faibles. Ces derniers sont parfois tellement faibles au final qu'ils peuvent nécessiter des détecteurs capables de réaliser du comptage de photons. Même si les détecteurs et leur chaîne de lecture présentent un très faible bruit, il peut s'avérer indispensable d'utiliser dans ce cas des détecteurs amplifiés. Dans tous les cas, les temps d'observation peuvent être importants, de sorte que les courants d'obscurité doivent être très faibles, ce qui impose la plupart du temps de refroidir les détecteurs. Notons toutefois que le besoin de filtrage est un peu moindre dans le cas de spectroscopie puisque la lumière visible est en partie écartée par dispersion.

Un autre besoin fréquent est celui de disposer de détecteurs de grands formats (de l'ordre de $2 \mathrm{k} * 2 \mathrm{k}$ voire $4 \mathrm{k}^{*} 4 \mathrm{k}$ pixels au pas de 10 à $\left.20 \mu \mathrm{m}\right)$. C'est particulièrement le cas pour les coronographes, en raison de la zone occultée correspondant au disque solaire. Pour les autres applications, ce besoin est également présent pour augmenter la résolution spatiale.

Pour les imageurs, certaines fonctionnalités peuvent s'avérer nécessaires (possibilité de réaliser du fenêtrage, disposer de temps d'intégration différents par zone... ).

\section{DÉTECTEURS UV : DIFFÉRENTES TECHNOLOGIES DISPONIBLES ET VOIES D'AMÉLIORATION}

On peut classer les différents détecteurs UV en trois grandes catégories : les détecteurs classiques en Silicium (CCD, APS), les détecteurs à large band-gap, et pour finir les détecteurs amplifiés.

\subsection{Détecteurs classiques à base de Silicium}

Les détecteurs classiques à base de Silicium (CCD et détecteurs APS) tirent avantage de leur forte maturité. Des larges formats sont disponibles en CCD mais également en APS. Pour diverses raisons (mise en œuvre, consommation, certaines fonctionnalités...), les APS (également appelés détecteurs CMOS) sont maintenant préférés aux CCD.

Les CCD restent toutefois largement utilisés : certes leur rendement quantique est faible dans l'UV, mais diverses solutions mises au point sur les CCD permettent d'obtenir in fine de bonnes performances. En effet, le développement de CCD amincis et utilisés en éclairement face arrière, permet d'obtenir un rendement quantique de l'ordre de 10 à $20 \%$ entre 100 et $400 \mathrm{~nm}$. L'utilisation combinée d'autres techniques sur ces CCD amincis (dépôt de Lumogen, dépôt d'une couche antireflet, technique du delta doping [1]) permet d'atteindre jusqu'à 40\% de rendement quantique entre 100 et $250 \mathrm{~nm}$ et $80 \%$ entre 250 et $400 \mathrm{~nm}$. Cependant, ces optimisations n'ont pas été mises au point à l'heure actuelle sur les APS (tout du moins en Europe), et des efforts de développement restent à faire en ce sens.

Même si certains APS peuvent présenter à l'heure actuelle un très faible bruit, les performances globales de bruit de ce type de détecteur associé aux chaîne de lecture ne permettent pas d'effectuer du comptage de photon voire de la détection de très faibles flux. Notons de plus que ce type de détecteur n’est pas solar-blind et nécessite donc la présence de filtres supplémentaires.

\subsection{Détecteurs à large band-gap}

Comme leur nom l'indique, ces détecteurs sont constitués de matériaux semi-conducteurs à large band gap. Ainsi, les photons du visible ne sont pas suffisamment énergétiques pour faire transiter un électron de la bande de valence à la bande de conduction par effet photo-électrique. De plus, le courant d'obscurité est théoriquement faible (ne nécessitant pas ou peu de refroidissement) et le rendement quantique peut être élevé si les détecteurs sont amincis. Deux types de matériaux sont particulièrement 
étudiés pour réaliser ce type de détecteur: le diamant et l'AlGaN. Toutefois, à l'heure actuelle, c'est principalement l'AlGaN qui est utilisé pour réaliser des détecteurs matriciels.

Ce type de détecteur présente cependant une faible maturité. En effet, la croissance d'AlGaN n'est pas aisée, et il existe des mauvaises adaptations avec les substrats utilisés. De ce fait, l'apparition de fortes dislocations entraîne la présence de courants d'obscurité plus importants que prévu. On peut aussi citer que les phénomènes de photoémission au niveau des électrodes sont responsables d'une réjection de la lumière visible moins bonne qu'espérée (on obtient tout de même une réjection de 4 ordres de grandeurs au-delà de $400 \mathrm{~nm}$ ). De plus, des difficultés d'amincissement de la face arrière pour une bonne efficacité autour de $100 \mathrm{~nm}$ sont rencontrées. Pour finir, tout comme les détecteurs classiques en Silicium, ce type de détecteur ne permet pas de réaliser de la détection de très faibles flux.

Même si la maturité de cette technologie reste faible, on peut toutefois citer deux réalisations de matrice à l'heure actuelle, de format 320*256 au pas de $30 \mu \mathrm{m}:$ l'une par BAE (US) [2] et l'autre par TRT (France) [3]. Toutefois, la possibilité de développer des matrices performantes de plus grand format reste à démontrer.

\subsection{Détecteurs amplifiés}

Comme nous l'avons vu précédemment, les détecteurs amplifiés sont indispensables pour la détection de très faibles flux voire le comptage de photons. Différentes technologies existent, dont principalement les tubes à GMC («Galettes de MicroCanaux»), les EBCCD/EBAPS (EB pour «Electron Bombarded»), et la technologie à base de photodiodes à avalanche (APD). Parmi ces technologies, la première est la plus utilisée dans les missions spatiales UV (la technologie APD est très prometteuse, mais elle souffre encore d'imperfections qui rendent son utilisation spatiale difficile). En plus de sa bonne maturité, la technologie à GMC possède un avantage technique sur les deux autres qui est d'être «solar-blind». En effet, les photocathodes utilisées permettent en général de rejeter la lumière visible avec une bonne efficacité (jusqu'à $10^{9}$ ). De plus, ces détecteurs peuvent être de taille relativement grande (plusieurs dizaines de mm de diamètre en surface utile) et peuvent être accouplés à des CCD ou APS de grand format. En revanche, on peut citer quelques désavantages relatifs à ce type de détecteur :

- la résolution spatiale est dégradée par les distances entre les différents éléments du tube (contraste typique de l'ordre de $50 \%$ @ 20 pl/mm), même si le diamètre des $\mu$ canaux est petit (de l'ordre de quelques $\mu \mathrm{m}$ )

- la mise en œuvre est délicate (présence de haute tension, le tube doit être sous vide)

- le rendement quantique est faible (de l'ordre de 20 à $40 \%$ pour des photocathodes CsBr ou CsI autour de $150 \mathrm{~nm}$ [4] et une transmission des fenêtres d'entrée de l'ordre de 50\%)

On peut toutefois souligner que des travaux sont actuellement en cours pour améliorer le rendement de détection en utilisant des nouveaux matériaux pour les photocathodes. L'AlGaN permettrait notamment d'atteindre de l'ordre de $70 \%$ de rendement quantique entre 100 et $200 \mathrm{~nm}$, avec toutefois une coupure du visible moins efficace qu'avec des photocathodes en CsI.

Les EBCCD/EBAPS souffrent d'une faible maturité, mais cependant certains progrès et la possibilité d'utiliser des APS delta dopés pourraient rendre ces détecteurs très attractifs (fort rendement quantique, bonne résolution spatiale). Toutefois, on dispose de peu de visibilité et de disponibilité de ce type de détecteur en Europe, la technologie étant brevetée.

\subsection{Comparaison des différentes technologies}

Le Tableau 1 résume les différents avantages et inconvénients des technologies citées précédemment :

On se rend compte que les CCD et APS sont les détecteurs de choix pour les applications à flux suffisamment élevés. Les détecteurs à large band-gap pourraient avantageusement les remplacer, leur maturité reste cependant médiocre à l'heure actuelle (performances à optimiser, réalisation de détecteurs grand format...). De sorte que les efforts sont à poursuivre pour une utilisation future possible à long 
Tableau 1. Performances des différentes technologies.

\begin{tabular}{|c|c|c|c|c|c|c|}
\hline & $\begin{array}{l}\text { Rendement } \\
\text { quantique }\end{array}$ & Résolution & $\begin{array}{l}\text { Comptage } \\
\text { photon/Très } \\
\text { faibles flux }\end{array}$ & $\begin{array}{l}\text { Réjection } \\
\text { du visible }\end{array}$ & Maturité & $\begin{array}{l}\text { Mise } \\
\text { en } \\
\text { oeuvre }\end{array}$ \\
\hline $\begin{array}{ll}\text { APS } & \& \\
\text { CCD } & \end{array}$ & $\begin{array}{l}\text { Jusqu'à } 70 \% \text { en } \\
\text { utilisation face } \\
\text { arrière }\end{array}$ & $\begin{array}{l}\text { Très } \\
\text { bonne }\end{array}$ & $\begin{array}{l}\text { Faibles } \\
\text { flux/flux } \\
\text { modérés } \\
\text { seulement }\end{array}$ & Absente & $\begin{array}{l}\text { Excellente } \\
\text { (excepté les } \\
\text { traitements } \\
\text { spécifiques } \\
\text { UV sur APS) }\end{array}$ & + \\
\hline $\begin{array}{ll}\text { Tubes } & \text { à } \\
\text { GMC } & \end{array}$ & $\begin{array}{l}\text { De qqs } \\
\% \text { à } 35 \% \text { selon } \\
\text { photocathode }\end{array}$ & Dégradée & OUI & $\begin{array}{l}\text { Très } \\
\text { bonne }\end{array}$ & Bonne & - \\
\hline $\begin{array}{l}\text { Détecteurs } \\
\text { à large } \\
\text { band-gap }\end{array}$ & $\begin{array}{l}\text { Qqs } \% \text { de } 100 \text { à } \\
250 \mathrm{~nm} \\
\text { De } 10 \text { à } 40 \% \text { de } \\
250 \text { à } 300 \mathrm{~nm}\end{array}$ & $\begin{array}{l}\text { Très } \\
\text { bonne }\end{array}$ & $\begin{array}{l}\text { Impossible à } \\
\text { l'heure } \\
\text { actuelle }\end{array}$ & Bonne & Mauvaise & $+\grave{a}++$ \\
\hline
\end{tabular}

terme. Pour les très faibles flux, les détecteurs amplifiés comme les tubes à GMC restent indispensables. De plus, les progrès en cours permettent d'atteindre des rendements quantiques satisfaisants. Cependant, la résolution spatiale reste dégradée, et ce malgré l'utilisation de $\mu$ canaux de faible diamètre.

Le Tableau 2 résume les détecteurs préconisés en fonction des différentes applications et de leurs besoins ainsi que des performances de chaque technologie.

Tableau 2. Détecteurs et applications possibles.

\begin{tabular}{|l|l|l|l|l|}
\hline Application: & $\begin{array}{l}\text { Observation solaire: } \\
\text { Coronographes }\end{array}$ & $\begin{array}{l}\text { Observation solaire: } \\
\text { Imageurs du disque }\end{array}$ & Planétologie & $\begin{array}{l}\text { Exploration } \\
\text { extra-galactique }\end{array}$ \\
\hline Détecteur: & $\begin{array}{l}\text { Détecteur amplifié } \\
\text { (car très faible flux) } \\
\text { et large format }\end{array}$ & $\begin{array}{l}\text { APS grand format } \\
\text { Eventuellement } \\
\text { détecteur } \\
\text { à large band-gap si } \\
\text { maturité suffisante }\end{array}$ & $\begin{array}{l}\text { Principalement } \\
\text { détecteur amplifié. } \\
\text { Parfois APS } \\
\text { possible }\end{array}$ & $\begin{array}{l}\text { Principalement } \\
\text { détecteur } \\
\text { amplifié }\end{array}$ \\
& & & \\
\hline
\end{tabular}

\section{DÉVELOPPEMENTS RÉCENTS INITIÉS PAR LE CNES ET PERSPECTIVES}

\subsection{Tube à GMC Lyman- $\alpha$}

Un tube à GMC a été développé par PHOTONIS (France) dans le cadre d'une action de R\&T menée par le CNES (R\&T pour «Recherche et Technologies») en collaboration étroite avec l'IAS (Orsay). Le but de cette R\&T étant de développer un tube optimisé pour la longueur d'onde à Lyman- $\alpha$ tout en tirant partie des faibles diamètres de $\mu$ canaux disponibles chez PHOTONIS afin d'améliorer la résolution spatiale.

Le rendement quantique obtenu est relativement bas car le dépôt de photocathode (CsI) directement sur GMC n'est pas aisé, ce dernier ayant été fait afin de gagner en résolution. De plus, l'utilisation de $\mu$ canaux de petite taille n'a pas permis un gain significatif en résolution. Celle-ci serait, à partir d'une certaine taille de $\mu$ canaux, limitée par la distance entre la galette et l'écran de Phosphore que l'on peut difficilement réduire. Les principales caractéristiques et performances de ce tube sont résumées dans le tableau 3.

Les performances obtenues sont toutefois satisfaisantes vis à vis de l'état de l'art. De plus, cette action de R\&T a permis d'appréhender les limitations actuelles de ce type de détecteur avec ce type 


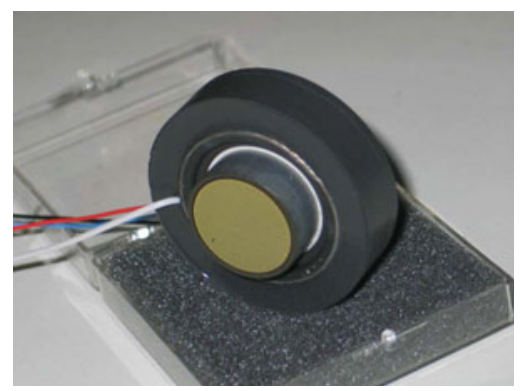

Figure 1. Tube développé par PHOTONIS.

Tableau 3. Principales caractéristiques et performances du tube développé par PHOTONIS dans le cadre de la R\&T CNES.

\begin{tabular}{|l|l|}
\hline Dimensions & $\begin{array}{l}25 \mathrm{~mm} \text { de diamètre en surface utile } \\
\mu \text { canaux de } 4.5 \mu \mathrm{m} \text { de diamètre }\end{array}$ \\
\hline Fenêtre d'entrée & $\begin{array}{l}\text { MgF2 } \\
\text { Transmission de } \sim 30 \% \text { à Ly- } \alpha \\
\text { Présence d'une grille repousseuse pour } \\
\text { augmenter le rendement quantique }\end{array}$ \\
\hline Format de sortie & Ecran Phosphore P43 + fibres optiques \\
\hline Photocathode & $\begin{array}{l}\text { CsI, dépôt sur la galette } \\
\sim 20 \% \text { de QE à Ly- } \alpha\end{array}$ \\
\hline Rendement quantique global à Ly- $\alpha$ & $\sim 6 \%$ \\
\hline Réjection visible & $>10^{9}$ \\
\hline Gain Global (photons sortie/photons entrée, QE & Ajustable (HT GMC) \\
inclus) à Ly- $\alpha$ & $\sim 10000$ pour $1100 \mathrm{~V}$ \\
\hline Résolution spatiale & $50 \%$ de contraste à $25 \mathrm{pl} / \mathrm{mm}$ \\
\hline
\end{tabular}

de photocathode (difficultés à améliorer la résolution, orientation vers d'autres types de photocathode comme l'AlGaN pour améliorer le rendement quantique... ). Pour finir, la mesure de résolution n'est pas simple et il se peut que celle-ci ait été sous-estimée. En effet, une mesure avec tube couplé à un détecteur aurait été plus précise. Cependant, pour des raisons purement programmatiques et de priorité technologique associée, l'action prévue de couplage de ce tube avec un APS a été annulée.

\subsection{Matrice AIGaN optimisée pour détection deep-UV}

Dans le but d'évaluer plus précisément les performances atteignables dans l'UV lointain (autour de 100 à $200 \mathrm{~nm}$ ) par les détecteurs AlGaN matriciels, une action de R\&T est menée par le CNES avec TRT. Outre les aspects performances, le but de cette R\&T est la mise au point d'un process d'amincissement de la face arrière satisfaisant et reproductible.

Une matrice de $320 * 256$ diodes schottky au pas de $30 \mu \mathrm{m}$ a servi de base à ce développement. La croissance de l'AlGaN est réalisée sur substrat Silicium. La matrice est ensuite hybridée sur un circuit de lecture via des billes d'Indium. Le substrat et les couches graduelles d'AlGaN sont alors gravés par ICP (Inductively Coupled Plasma») afin d'atteindre la zone active et d'améliorer le rendement quantique dans l'UV profond. Une structure en nid d'abeille est réalisée afin de garantir une bonne rigidité de membrane et d'éviter l'apparition de «cracks ».

Les matrices développées sont fonctionnelles et présentent moins de $2 \%$ de pixels défectueux. L'apparition de cracks a été constatée dans certains cas, mais un process de gravure garantissant leur 


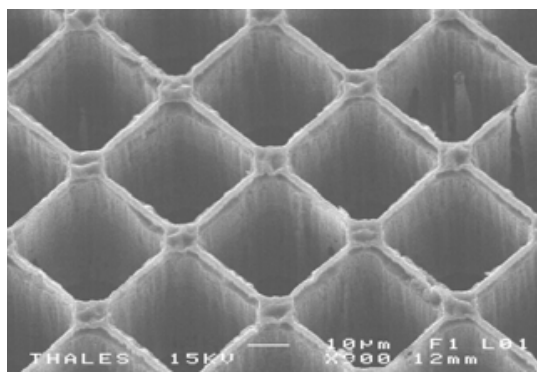

a)

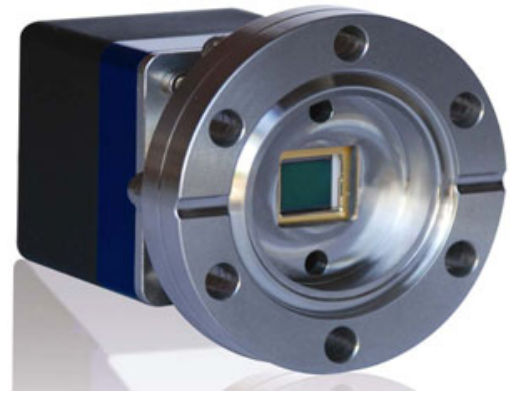

b)

Figure 2. (a) Vue de la face arrière de la matrice AlGaN après amincissement (structure en nid d'abeille) (b) Matrice et électronique intégrée dans une caméra pour mesures sous vide des performances électro-optiques.

absence a été identifié. Le bruit de lecture mesuré est satisfaisant (de l'ordre de 150 e- rms correspondant au bruit de lecture propre au circuit et au bruit en kTC de la capacité détecteur qui est de 20fF) et le courant d'obscurité est inférieur au fA à l'ambiante.

Le rendement quantique mesuré de 250 à $400 \mathrm{~nm}$ montre une bonne réjection de la lumière visible (de l'ordre de $10^{4}$ à partir de $400 \mathrm{~nm}$ ) et un rendement quantique proche UV de l'ordre de $10 \%$ (cf. figure 3).

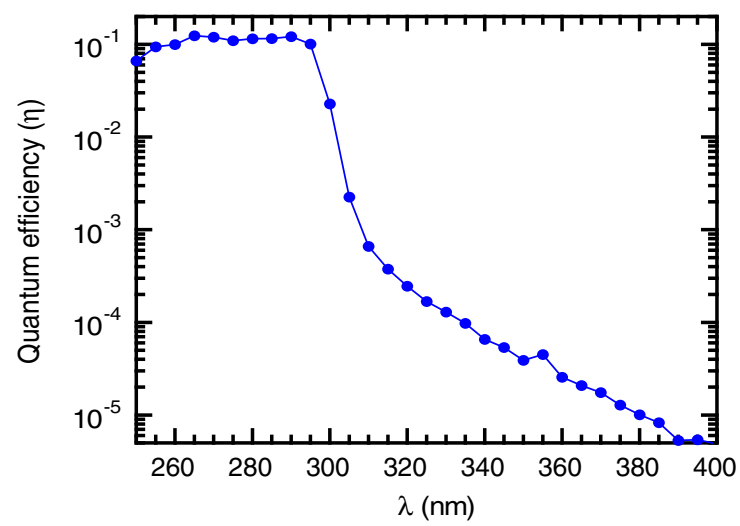

Figure 3. Rendement quantique mesuré entre 250 et $400 \mathrm{~nm}$.

En revanche, ce dernier tombe à quelques \% seulement entre 100 et $200 \mathrm{~nm}$. Il faut toutefois signaler que le rendement quantique intrinsèque au détecteur est deux fois plus élevé, puisque la structure en nid d'abeille donne un facteur de remplissage de $40 \%$ seulement. L'utilisation de $\mu$ lentilles pourrait donc a priori permettre de doubler ce $\mathrm{QE}$, à condition qu'une telle technologie soit possible dans ce domaine de longueurs d'onde.

Les pré-évaluations technologiques prévues dans le cadre de cette R\&T (life test, cyclage thermique) seront réalisées prochainement.

Globalement, on pourra retenir que les performances en UV lointain restent décevantes, mais que le process de gravure mis au point est satisfaisant car reproductible et permettant d'éviter la présence de cracks. De plus, des voies d'amélioration ont été identifiées et pourraient faire l'objet d'actions 
ultérieures :

- amélioration du matériau par l'emploi de nouvelles couches d'épitaxie

- reprise du process de gravure pour amélioration du QE dans l'UV lointain (dopages, profondeurs de gravure, combinaison éventuelle avec une gravure chimique...)

- amélioration de la réjection du visible par l'utilisation d'architectures de pixels différentes permettant de réduire le phénomène de photo-émission

- réalisation d'une matrice $620 * 512$ pixels au pas de $15 \mu \mathrm{m}$, permettant d'étudier la possibilité de réaliser de plus grands formats, et de réduire le bruit de lecture en réduisant la taille du pixel

\subsection{Perspectives}

Une roadmap CNES «Détection UV»a été établie en 2008 en prenant en compte les aspects programmatiques et les développements nécessaires pour satisfaire les besoins des futures missions spatiales.

Le premier objectif visé consiste à évaluer les possibilités d'amélioration des performances obtenues sur les premières réalisations de détecteurs $\mathrm{AlGaN}$ afin de statuer sur la crédibilité de leur utilisation possible à moyen/long terme.

Ensuite, des essais de portage des techniques d'optimisation du rendement quantique dans l'UV pour les CCD sont envisagés sur des détecteurs APS.

Ces deux actions ne sont pas à l'heure actuelle programmées de manière formelle et seront soumises à décision de lancement fin 2010/début 2011.

\section{Références}

[1] http://www.stsci.edu/stsci/meetings/space_detectors/pdf/nikzad.pdf.

[2] M. B. Reine et al. , Solar-blind AlGaN 256*256 p-i-n detectors and focal plane arrays, Proc. Of SPIE Vol. 6119611901 (2006).

[3] J. L. Reverchon et al., Status of backthinned AlGaN based focal plane arrays for deep-UV imaging, International Conference on Space Optics (2010).

[4] B. K. Singh et al. , CsBr and CsI UV photocathodes: new results on quantum efficiency and aging, IEEE Transactions on electron devices, Nucl. Instr. \& Meth. A454 pp. 364-378 (2000). 